\title{
A National Evaluation of Surgeon Experiences in Telemedicine for the Care of Hernia and Abdominal Core Health Patients
}

\author{
Vahagn C. Nikolian ${ }^{1}$ Mudassir Akhter ${ }^{1} \cdot$ Emaad J. Iqbal $^{2}$ (1) $\cdot$ Thomas Sutton $^{1} \cdot$ Ashraf Samhan $^{1} \cdot$ \\ Sean B. Orenstein ${ }^{1} \cdot$ Michael J. Rosen ${ }^{3} \cdot$ Benjamin K. Poulose $^{4}$
}

Accepted: 11 September 2021 / Published online: 3 October 2021

This is a U.S. government work and not under copyright protection in the U.S.; foreign copyright protection may apply 2021

\begin{abstract}
Background Surgeons are increasingly utilizing telemedicine to provide perioperative services to patients. Safety, satisfaction, and feasibility of these programs in general populations have been established, but it is unclear how telemedicine can be integrated into subspecialty care. We report results of a national survey related to telehealth practices among members of the Abdominal Core Health Quality Collaborative (ACHQC).

Methods Survey responses were analyzed to determine current strategies in telemedicine utilization. Surgeon preferences, perceptions of validity, and identified barriers to implementation of telemedicine were assessed.

Results Forty surgeons within the ACHQC responded, with $90 \%$ of respondents reporting use of telemedicine to deliver perioperative care to patients with hernias and abdominal core health concerns. Surgeons appeared to be more comfortable managing preoperative patients with image-confirmed diagnoses of hernias. Surgeons were universally more comfortable delivering postoperative care via telemedicine. Connectivity, patient engagement, and reimbursement were identified as potential barriers to expansion of telemedicine. Seventy-eight percent of respondents reported that they would increase telemedicine utilization if current regulations were maintained in the future.

Conclusions This study found that hernia specialists are utilizing telemedicine at a higher rate than before the COVID-19 pandemic, with surgeons reporting interest in continued use of this modality beyond the pandemic. These findings suggest that future work in telemedicine optimization may improve the quality of care that can be delivered to patients with abdominal core health concerns.
\end{abstract}

Vahagn C. Nikolian

nikolian@ohsu.edu

1 Department of Surgery, Division of Gastrointestinal and General Surgery, Oregon Health \& Science University, Mail Code L 233A, 3181 SW Sam Jackson Park Road, Portland, OR 97239, USA

2 Department of Surgery, Columbia University Irving Medical Center, New York, NY, USA

3 Center for Abdominal Core Health, Cleveland Clinic Foundation, Cleveland, OH, USA

4 Center for Abdominal Core Health, Ohio State University, Columbus, OH, USA

\section{Introduction}

The coronavirus disease 2019 (COVID-19) pandemic has disrupted well-established practice models in healthcare [1]. In an attempt to preserve medical resources and reduce disease spread, hospital systems developed innovative workflows to allow for delivery of care, with an increased focus on the utilization of telemedicine and virtual care $[2,3]$. Though these technologies have been available for decades, they have been underutilized secondary to perceived barriers related to lack of direct patient contact, limitations in performing an adequate physical examination, and challenging reimbursement models [4]. Evidence suggests that telemedicine approaches can be accurate [5], 
cost-effective [6], safe [7], and associated with appropriate patient satisfaction [8].

For surgeons, the pandemic has forced many to significantly increase telemedicine utilization for a variety of patient interactions. This rapid implementation process has given the surgical community a snapshot of the current status of telemedicine evaluations. Though many institutions and stakeholders are enthusiastic about telemedicine, little is known about the role that this technology can have in specific surgical populations. The COVID-19 pandemic has given surgeons an opportunity to assess what patient populations may be best served with telemedicine platforms and which elements of telemedicine technology require further optimization.

Hernia repair is one of the most common operations performed in the USA $[9,10]$. Given the rapid increase in telemedicine utilization, it is necessary to evaluate provider experiences to guide future strategies in implementation and expansion of telemedicine for specific patient populations. To our knowledge, no studies have reviewed surgeon impressions of the utility of telemedicine evaluations for hernias and abdominal core health. In this study, we sought to assess utilization of telemedicine by surgeons within the Abdominal Core Health Quality Collaborative (ACHQC) for the management of patients with hernias and abdominal core health concerns.

\section{Material and methods}

In this survey-based study, a 27-question web-based survey (SurveyMonkey ${ }^{\mathrm{TM}}$; Portland, OR, USA) was developed to assess surgeon telehealth utilization during the COVID-19 pandemic. (Supplementary 1) The study population included all active members of the ACHQC, a non-profit organization with aims to improve outcomes and value in abdominal core and hernia patient care through data collection, analysis, and collaborative learning. The survey items evaluated current practices, assessed perceived obstacles and enablers of telemedicine implementation, and reviewed surgeon opinion of case types that were well suited for digital-based consultation. The survey collected quantitative and qualitative data. The anonymous, electronic questionnaire was sent to the 237 active members of the ACHQC in May 2020. The survey took an estimated 10 min to complete. Reminders were sent two weeks following the initial email. All survey responses were deidentified.

Basic surgeon demographic information was obtained using the survey. We sought to understand how surgeons utilized telemedicine during the COVID-19 Pandemic. Survey questions focused on surgeon experience, prior exposure to telemedicine, the impact of COVID-19 on clinic volume, and the modalities used to perform digital patient care. We focused on understanding factors specific to hernia care, along with responses about personal experiences taking care of patient with a variety of commonly encountered conditions. Surgeons were asked about their comfort with evaluating patients in the pre- and postoperative period. Questions related to perceived barriers were addressed to inform future optimization. The results were detailed from the compiled outcomes. Chi-squared testing was utilized to evaluate survey answers by respondent demographic groups; the Yates correction for continuity was not utilized when calculating Chi-squared $P$ values. Data were analyzed using SPSS version 27 (IBM Corp, Armonk, NY). This research project was approved by Oregon Health \& Science University's Institutional Review Board.

\section{Results}

Between May 21, 2020 and June 7, 2020, the survey was distributed to 237 active members of the ACHQC, and 40 responses were recorded (16.9\% response rate). Demographics of respondents are reviewed and summarized in Table 1 . The majority of surgeons reported at least 8 years of practice as high-volume hernia surgeons $(77.5 \%)$ and worked in academic $(45 \%)$ or academically affiliated practice environments (32.5\%). All (100\%) surgeons reported that COVID-19 impacted their in-person clinic volume, with the majority (65\%) reporting that they were required to completely suspend in-person clinic evaluations.

\section{Telemedicine utilization prior to pandemic}

Most surgeons $(82.5 \%)$ reported that they had never utilized telemedicine prior to the pandemic. Similarly, $85 \%$ reported that they were unaware telemedicine modalities existing at their institution. Volume of telemedicine encounters was sparse, with only two (5\%) surgeons reporting more than one telemedicine evaluation per month in the pre-pandemic period. Of the seven pre-pandemic telemedicine utilizers, services provided were centered around evaluation of established patients $(57 \%)$ and immediate postoperative care $(86 \%)$. Only three surgeons (7.5\%) reported using telemedicine for new patient encounters. No clear trends were established for billing of patient encounters in the pre-pandemic period.

\section{Telemedicine utilization during the pandemic}

Surgeons increased telemedicine utilization during the COVID-19 pandemic, with $90 \%$ of surgeon respondents 
Table 1 Demographics of respondents $(n=40)$

\begin{tabular}{ll}
\hline Surgeon-reported characteristics & Total (\%) \\
\hline Training background & $19(47.5 \%)$ \\
Board certified general surgeon with no fellowship training & $19(47.5 \%)$ \\
Board certified general surgeon with fellowship training in minimally invasive surgery (MIS) & $2(5 \%)$ \\
Board certified general surgeon with other fellowship training & \\
Number of years in practice (years) & $4(10 \%)$ \\
$1-3$ & $5(12.5 \%)$ \\
$4-7$ & $12(30 \%)$ \\
$8-15$ & $19(47.5 \%)$ \\
$>15$ & $18(45 \%)$ \\
Clinical practice environment & $13(32.5 \%)$ \\
Academic & $9(22.5 \%)$ \\
Academic affiliated & $6(15 \%)$ \\
Private practice & $7(17.5 \%)$ \\
Surgeon reported proportion of cases that are hernia related & $13(32.5 \%)$ \\
$<25 \%$ & $14(35 \%)$ \\
$25-50 \%$ & \\
$51-75 \%$ & $75 \%$
\end{tabular}

reporting use of these services for patient care compared to $17.5 \%$ pre-pandemic $(P<0.001$, Table 2$)$. A variety of applications were used by surgeons to connect with patients, with Zoom (San Jose, CA) being the most utilized (50\% of respondents) and most preferred (31\% of respondents). Frequency of telemedicine evaluations increased during the pandemic, with $69 \%$ of surgeons reporting more than 10 digital patient encounters per month. In contrast to the pre-pandemic period, services spanned the full spectrum of perioperative care, with nearly all respondents reporting use of telemedicine for established (94\%), new patient encounters (81\%), and postoperative care $(94 \%)$. The majority (83\%) of surgeons used time-based billing for encounters. Notably, patterns of telemedicine services utilization differed by respondent demographics: $95 \%$ ( $n=17$ ) of MIS-fellowship-trained respondents reported using telehealth for new patient encounters, compared to $66 \%(n=12)$ of non-MIS-trained respondents $(P=0.035)$; identical results were noted for respondents in academic compared to private practice settings. Ninety-five percent $(n=19)$ of respondents in practice for $<15$ years reported utilization of telehealth for new patient evaluations, compared to $62.5 \%(n=10)$ of respondents in practice for $>$ 15 years $(P=0.014)$. There were no significant differences in telehealth utilization for established or postoperative visits by practice duration, practice setting, or fellowship ( $P>0.1$ for all); proportion of clinical volume constituted by abdominal core health was not associated with utilization of telehealth for new, established, or postoperative patients.

\section{Hernia care rendered using telemedicine services}

We assessed hernia-specific care that surgeons provided using telemedicine. (Table 3) Seventy percent of surgeons reported using telemedicine in the pre- and postoperative setting. Surgeons felt comfortable evaluating the following diagnoses virtually: primary inguinal hernia (79\% of respondents), primary ventral (75\% of respondents), and incisional hernias without loss of domain $(68 \%$ of respondents). Diagnoses-related enterocutaneous fistulae (11\% of respondents), incisional hernias with loss of domain (25\% of respondents), and groin pain (29\% of respondents) were less commonly managed using telemedicine strategies.

The most common case types that were evaluated in the postoperative setting included minimally invasive surgical (MIS) repair of groin hernias (82\%), MIS repair of primary ventral hernias $(75 \%)$, and open repair of groin hernias (61\%). Respondents in academic practice settings were less likely to utilize telemedicine services postoperatively for patients undergoing surgery for groin pain $(0 \%, n=0)$ than private practitioners $(33 \%, n=5 ; P=0.01)$, with similar results for respondents that were MIS-fellowship trained (0\% versus $36 \%$; $P=0.006$ ). Respondents with MIS-fellowship training $(39 \%, n=7)$ were non-significantly less likely to report utilizing telemedicine services for the 
Table 2 Surgeon reported telemedicine utilization during the pandemic

\begin{tabular}{ll}
\hline Responses (Total 36) & $n(\%)$ \\
\hline Platforms used for video based & \\
Zoom & $18(50 \%)$ \\
EMR-based application & $9(25 \%)$ \\
Doxy.me & $9(25 \%)$ \\
Doximity & $8(22 \%)$ \\
Facetime & $8(22 \%)$ \\
Other* & $9(25 \%)$ \\
Preferred platform & \\
Zoom & $11(31 \%)$ \\
Doximity & $7(19 \%)$ \\
EMR-based application & $5(14 \%)$ \\
Doxy.me & $4(11 \%)$ \\
Other $* *$ & $4(11 \%)$ \\
Estimated video-based evaluation performed per month \\
$<1$ & $3(8 \%)$ \\
1-10 & $8(22 \%)$ \\
$11-20$ & $12(33 \%)$ \\
$>$ 20 & $13(36 \%)$ \\
Services provided to patients & \\
Evaluation of new patients & $29(81 \%)$ \\
Immediate postoperative care & $34(94 \%)$ \\
Evaluation of established patients & $34(94 \%)$ \\
Time-based billing & \\
Bundled payments related to operation & $31(83 \%)$ \\
No billing for telephone encounter & $11(28 \%)$ \\
\hline Other Updocs & $8(22 \%)$ \\
\hline
\end{tabular}

*Other: Updocs (2), Amwell (2), Android (1), E-Visit (1), Duo (1), Webex (1), Vidyo (1)

** No other choices offered/nothing to compare (3), Vidyo (1)

postoperative evaluation of open inguinal hernia repair versus non-fellowship-trained respondents $(71 \%, n=10$; $P=0.067)$. There were no other trends in utilizing telemedicine postoperatively for open/MIS repair of inguinal hernias, surgery for groin pain, open/MIS repair of epigastric/umbilical/flank hernias, or incisional hernias with or without loss of domain by practice setting, duration, fellowship training, or practice volume $(P>0.2$ for all).

\section{Perceptions regarding the future of telemedicine}

We next assessed surgeon perceptions on the viability of telemedicine evaluations in a variety of case scenarios and compared them by respondent demographics (Table 4). The majority of surgeons believed that video-based evaluation was a viable option for patients presenting with imaging-confirmed diagnoses of primary ventral $(81 \%)$, inguinal (72\%), and incisional/recurrent hernias (64\%). Surgeons felt that these suspected diagnoses without imaging were less viable for preoperative telehealth evaluation (39\%, 33\%, and $26 \%$, respectively). A minority of surgeons believed groin pain $(6 \%)$ and enterocutaneous fistulae $(23 \%)$ could be appropriately managed with a preoperative telehealth evaluation. Almost universally, surgeons felt that telehealth evaluations were more viable in the postoperative setting. Surgeons believed in the viability of video-based evaluation of postoperative patients following MIS groin hernia repair (92\%), open repair of groin hernias (89\%), and MIS repair of ventral and incisional hernias without myofascial release $(81 \%)$. The majority (92\%) of surgeons responded that video-based postoperative care was efficient.

There were significant differences in the aforementioned metrics by practice setting, number of years in practice, and MIS-fellowship training (Table 4). Broadly, respondents in practice for $<15$ years, those practicing in an academic setting, and those with MIS-fellowship training were more likely to perceive telehealth as a viable platform for both preoperative and postoperative care across the spectrum of abdominal core health operations and diagnoses. Respondent perceptions on the pre- and postoperative use of telehealth services did not significantly differ by clinical practice volume, except for in the evaluation of patients with clinically diagnosed incisional or recurrent hernias ( $0 \%$ vs $37.5 \%$ perceiving utility for respondents with $<$ $50 \%$ vs $>50 \%$ practice volume composed of abdominal core health cases; $P=0.014$ ), and in the postoperative evaluation of open/MIS repair of ventral/incisional hernias with myofascial release (25\% vs $66.7 \%$ for respondents with $<50 \%$ vs $>50 \%$ practice volume composed of abdominal core health cases; $P=0.018$ ). Respondents were not significantly different in their perceived trajectory of telehealth use after the pandemic, regardless of practice setting, practice duration, clinical volume, or MIS training (all $P>0.1$ ).

\section{Perceived barriers related to telemedicine expansion}

The final portion of the survey focused on assessing the perceived barriers associated with telemedicine expansion. The findings of these results are presented in Table 5 . Though most surgeons found the barriers queried to be minor or moderate, a higher proportion of respondents felt that connectivity $(31 \%)$, patient engagement $(28 \%)$, and cost/reimbursement (22\%) were significant barriers to effective implementation of telemedicine evaluations. Staffing, lack of medical records, provider/institutional resistance, and scalability were considered to be minor and/ or moderate barriers to implementation by more than $90 \%$ of respondents. Overall, $78 \%$ of surgeons reported that they 
Table 3 Hernia care rendered using telemedicine

\begin{tabular}{ll}
\hline Responses (Total 28) & $n(\%)$ \\
\hline Patient population evaluated preoperatively & $22(79 \%)$ \\
Primary Inguinal Hernias & $21(75 \%)$ \\
Primary Ventral Hernias & $19(68 \%)$ \\
Incisional Hernias without loss of domain & $11(39 \%)$ \\
Recurrent Inguinal Hernias & $8(29 \%)$ \\
Groin Pain & $7(25 \%)$ \\
Incisional Hernias with loss of domain & $3(11 \%)$ \\
Enterocutaneous fistula & \\
Patient population evaluated postoperatively & $23(82 \%)$ \\
MIS repair of groin hernias & $21(75 \%)$ \\
MIS repair of primary ventral hernias & $17(61 \%)$ \\
Open repair of groin hernias & $16(57 \%)$ \\
Open repair of incisional hernias without loss of domain & $14(50 \%)$ \\
Open repair of primary ventral hernias & $9(32 \%)$ \\
Open repair of incisional hernia with loss of domain & $5(18 \%)$ \\
Surgery for groin pain &
\end{tabular}

would increase telemedicine utilization if the emergency regulatory measures were to be maintained in the postpandemic period. There were no significant differences in perceived barriers to telemedicine expansion between respondent practice settings, practice duration, fellowship training, or clinical volume of abdominal core health cases $(P>0.1$ for all).

\section{Discussion}

In this study, the majority of respondents utilized telemedicine approaches during the COVID-19 pandemic, despite few reporting prior experience. Surgeons reported telemedicine's efficacy for preoperative evaluation of patients presenting with imaging-confirmed hernia diagnoses. For postoperative evaluation, surgeons reported comfort with evaluation of low-complexity hernia repairs via telemedicine encounters. The majority of surgeons anticipate an increase in telemedicine utilization if emergency regulations are maintained in the post-pandemic period. We were encouraged that barriers to implementation were perceived as minor to moderate inconveniences for most respondents.

This study is the first to assess telemedicine utilization among subspecialty surgeons. Prior studies have evaluated patient reported experiences [11], clinical outcomes [12], and the financial implications [13] of telemedicine, but lacked the granularity necessary to understand how telemedicine approaches can be utilized in various clinical scenarios and phases of care. We were encouraged to identify trends in specific case types that appear to be more conducive to a virtual visit format. In particular, surgeons appeared to have greater degrees of comfort associated with evaluating patients with image-confirmed diagnoses. It appears that surgeons were universally more comfortable with postoperative evaluation of patients, irrespective of the type of hernia or operation performed. Surgeons may have favored postoperative visits for a variety of reasons, including having an established relationship with patients, low complication rates among the case types evaluated, and a greater degree of evidence regarding the safety of postoperative telemedicine evaluations $[14,15]$. The description of surgeon perspective of which patients are appropriate for telemedicine evaluation is critical. This allows development of triage criteria for administrative staff to place patients into the appropriate evaluation modality. Future studies need to assess the accuracy of preoperative evaluations performed in the digital setting through prospective studies that evaluate the feasibility, efficacy, and safety of telemedicine strategies for different patient populations.

We also assessed the financial advantages and disadvantages of delivering care via telemedicine. From a surgeon perspective, we reviewed reimbursement strategies to determine the impact of the recent emergency interventions by CMS to establish reimbursement parity. Prior to the COVID-19 pandemic, reimbursement was available for a small proportion of preoperative services rendered in designated clinic locations or rural patient populations $[16,17]$. As a result, most surgeons only implemented telemedicine in postoperative care, where reimbursement was based on bundled payments. Our respondents reaffirmed that maintenance of emergency measures taken by CMS to establish payment parity in the post-pandemic period would increase telemedicine utilization. Though we did not evaluate patient experiences in this study, others have evaluated cost related to travel and missed work, demonstrating that virtual visits can be cost-effective means for patients to be evaluated $[18,19]$. Further studies should examine financial implications for other stakeholders, including institutions who would have less facility costs associated with virtual care.

Respondents cited concerns related to patient engagement and connectivity. Surgeons participating in our survey used a variety of platforms to connect with patients. Currently, HIPAA regulations have been paused to facilitate telemedicine assessments, but these temporary regulatory changes stand to convert back to pre-pandemic approaches. Recent work by Kemp et al. [14] demonstrated that no-show rates in telemedicine clinics are higher in African-American and single parent households. Studies evaluating patient engagement with electronic medical record-based applications and smart phones will need to be 
Table 4 Respondent Perceptions of Viability of Video-based evaluations by Diagnosis, Procedure, and Phase of Care

\begin{tabular}{|c|c|c|c|c|c|c|c|c|c|}
\hline & \multicolumn{3}{|c|}{ Practice duration, years } & \multicolumn{3}{|c|}{ Practice setting } & \multicolumn{3}{|c|}{ Fellowship training } \\
\hline & $\begin{array}{l}<15 \\
(n=19) \\
n(\%)\end{array}$ & $\begin{array}{l}>15 \\
(n=17) \\
n(\%)\end{array}$ & $\begin{array}{l}P \\
\text { Value }\end{array}$ & $\begin{array}{l}\text { Academic } \\
(n=16) \\
n(\%)\end{array}$ & $\begin{array}{l}\text { Private } \\
(n=20) \\
n(\%)\end{array}$ & $\begin{array}{l}P \\
\text { Value }\end{array}$ & $\begin{array}{l}\text { MIS } \\
(n=16) \\
n(\%)\end{array}$ & $\begin{array}{l}\text { None/ } \\
\text { Other } \\
\quad(n=20) \\
n(\%)\end{array}$ & $P$ Value \\
\hline $\begin{array}{l}\text { Perceived viability for new patient } \\
\text { evaluations }\end{array}$ & $15(78.9)$ & $5(29.4)$ & 0.003 & $12(75)$ & $8(40)$ & 0.036 & 13 (81.3) & $7(35)$ & 0.006 \\
\hline Imaging-confirmed inguinal hernia & $18(94.7)$ & $8(47.1)$ & 0.001 & $15(93.8)$ & $11(55)$ & 0.010 & $\begin{array}{l}16 \\
(100)\end{array}$ & $10(50)$ & $<0.001$ \\
\hline Clinically diagnosed inguinal hernia & $8(42.1)$ & $4(23.5)$ & 0.238 & $8(50)$ & $4(20)$ & 0.058 & $6(37.5)$ & $6(30)$ & 0.635 \\
\hline Groin pain* & $1(5.3)$ & $1(6.3)$ & 0.935 & $1(6.3)$ & $1(5.2)$ & 0.871 & $1(6.3)$ & $1(5.2)$ & 0.871 \\
\hline $\begin{array}{l}\text { Clinically diagnosed epigastric/umbilical } \\
\text { hernia }\end{array}$ & $10(52.6)$ & $4(23.5)$ & 0.074 & $8(50)$ & $6(30)$ & 0.221 & $8(50)$ & $6(30)$ & 0.221 \\
\hline $\begin{array}{l}\text { Imaging-confirmed epigastric/umbilical/ } \\
\text { flank hernia }\end{array}$ & $18(94.7)$ & $11(64.7)$ & 0.023 & $15(93.8)$ & $14(70)$ & 0.074 & $\begin{array}{l}16 \\
(100)\end{array}$ & $13(65)$ & 0.008 \\
\hline $\begin{array}{l}\text { Clinically diagnosed incisional/recurrent } \\
\text { hernias* }\end{array}$ & $6(31.6)$ & $3(18.6)$ & 0.335 & $6(37.5)$ & $3(15.8)$ & 0.121 & $6(37.5)$ & $3(15.8)$ & 0.121 \\
\hline $\begin{array}{l}\text { Imaging diagnosed incisional/recurrent } \\
\text { hernia }\end{array}$ & $14(73.7)$ & $9(52.9)$ & 0.196 & $12(75)$ & $11(55)$ & 0.214 & 13 (81.3) & $10(50)$ & 0.052 \\
\hline Enterocutaneous Fistula* & $5(26.3)$ & $3(18.8)$ & 0.532 & $5(31.3)$ & $3(15.8)$ & 0.244 & $5(31.3)$ & $3(15.8)$ & 0.244 \\
\hline $\begin{array}{l}\text { Perceived viability for postoperative } \\
\text { evaluations }\end{array}$ & $19(100)$ & $14(82.4)$ & 0.056 & $16(100)$ & $17(85)$ & 0.106 & $\begin{array}{l}16 \\
(100)\end{array}$ & $17(85)$ & 0.106 \\
\hline Open repair of inguinal/femoral hernia & $19(100)$ & $13(76.5)$ & 0.025 & $15(93.8)$ & $17(85)$ & 0.406 & $\begin{array}{l}16 \\
(100)\end{array}$ & $16(80)$ & 0.058 \\
\hline MIS repair of inguinal/femoral hernia & $19(100)$ & $14(82.4)$ & 0.056 & $15(93.8)$ & $18(90)$ & 0.686 & $\begin{array}{l}16 \\
(100)\end{array}$ & $17(85)$ & 0.106 \\
\hline Surgery for groin pain & $13(68.4)$ & $9(52.9)$ & 0.342 & $11(68.8)$ & $11(55)$ & 0.400 & 13 (81.3) & $9(45)$ & 0.027 \\
\hline $\begin{array}{l}\text { Open Repair of Ventral/Incisional Hernia } \\
\text { without myofascial release }\end{array}$ & $18(94.7)$ & $10(58.8)$ & 0.010 & $15(93.8)$ & $13(65)$ & 0.039 & $\begin{array}{l}15 \\
(93.8)\end{array}$ & $13(65)_{-}$ & 0.039 \\
\hline $\begin{array}{l}\text { MIS Repair of ventral/incisional hernias } \\
\text { without myofascial release }\end{array}$ & $18(94.7)$ & $11(64.7)$ & 0.023 & $15(93.8)$ & $14(70)$ & 0.074 & ${ }^{15}(93.8)$ & $14(70)$ & 0.074 \\
\hline $\begin{array}{l}\text { Open/MIS repair of ventral/incisional } \\
\text { hernia with myofascial release }\end{array}$ & $12(63.2)$ & $7(41.2)$ & 0.187 & $12(75)$ & $7(35)$ & 0.017 & 11 & $8(40)$ & 0.086 \\
\hline $\begin{array}{l}\text { Planned post-pandemic telehealth } \\
\text { utilization relative to pre-pandemic }\end{array}$ & & & 0.448 & & & 0.115 & & & 0.394 \\
\hline Decreased & $0(0)$ & $1(5.9)$ & & $0(0)$ & $1(5)$ & & $0(0)$ & $1(5)$ & \\
\hline Increased & $16(84.2)$ & $12(70.6)$ & & $15(93.8)$ & $13(65)$ & & 14 & $14(70)$ & \\
\hline No Change & $3(15.8)$ & $4(23.5)$ & & $1(6.3)$ & $6(30)$ & & $2(12.5)$ & $5(25)$ & \\
\hline
\end{tabular}

* Out of 35 respondents. Abbreviations: MIS = minimally invasive surgery

conducted to determine disparities in access to digital health [20].

While our research was able to glean granular data about the usage and perception of telemedicine among hernia surgeons, it has certain limitations. The targeted demographic was hernia and abdominal core health surgeons with results specific to hernia care. Our responding cohort and response rate was small; however, we were able to reach a highly experienced and specialized group of surgeons lending credibility to our findings. Further, the results lack generalizability to other specialties, and telemedicine may have a different dynamic as it relates to the workflow of other specialties (i.e., vascular/wound care clinics where in-person exam may be indispensable to decision making). Finally, the study population was limited to surgeons; further studies will be needed to assess patient and nursing staff perceptions and comfort with the use of telemedicine experiences before permanent changes are implemented. 
Table 5 Perceived barriers to utilization of telehealth

\begin{tabular}{llll}
\hline Responses (total 36) & $n(\%)$ & & \\
\cline { 2 - 4 } Perceived barriers to telehealth utilization & Minor & Moderate & Significant \\
\hline Patient engagement & $15(42 \%)$ & $10(28 \%)$ & $11(31 \%)$ \\
Connectivity & $15(42 \%)$ & $11 \%(28 \%)$ & $10(28 \%)$ \\
Cost/reimbursement & $18(50 \%)$ & $9(25 \%)$ & $8(22 \%)$ \\
Security & $23(64 \%)$ & $11(31 \%)$ & $3(11 \%)$ \\
Scalability & $22(61 \%)$ & $11(31 \%)$ & $2(6 \%)$ \\
Staffing & $23(64 \%)$ & $8(22 \%)$ & $1(3 \%)$ \\
Provider/institutional resistance & $27(75 \%)$ & $9(25 \%)$ & $0(0 \%)$ \\
Lack of medical records & $27(75 \%)$ &
\end{tabular}

Our study demonstrates that hernia surgeons utilized telemedicine approaches to ensure patients maintain access to perioperative care for elective hernia and abdominal core health concerns. Surgeons appear interested in implementing telemedicine strategies to provide care beyond the COVID-19 pandemic. We identify patient populations which may benefit from expansion of current regulations. Further, we identify barriers to telemedicine expansion that need to be addressed to optimize the experience for all stakeholders.

Supplementary Information The online version contains supplementary material available at https://doi.org/10.1007/s00268021-06332-9.

Acknowledgements We would like to acknowledge the contributions of Aileen Beckler, ACHQC Program Coordinator, for technical support in developing and distributing the survey to active members of the ACHQC.

\section{Declarations}

Conflict of interest Authors of this manuscript have each reviewed their conflict of interest/disclosure and funding/support relationships and deny any competing interests that potentially or inappropriately influence their work or conclusions in this manuscript.

\section{References}

1. Rose S (2020) Medical student education in the time of COVID19. JAMA 323(21):2131-2132

2. Gilson SF, Umscheid CA, Laiteerapong $N$ et al (2020) Growth of ambulatory virtual visits and differential use by sociodemographics at one urban academic medical center during the COVID-19 pandemic: retrospective analysis. JMIR Med Inform 8(12):e24544

3. Franzosa E, Gorbenko K, Brody AA et al (2021) "At home, with care": Lessons from New York City Home-based Primary Care practices managing COVID-19. J Am Geriatr Soc 69(2):300-306

4. Adler-Milstein J, Kvedar J, Bates DW (2014) Telehealth among US hospitals: several factors, including state reimbursement and licensure policies, influence adoption. Health Aff (Millwood) 33(2):207-215

5. Demartines N, Otto U, Mutter D et al (2000) An evaluation of telemedicine in surgery: telediagnosis compared with direct diagnosis. Arch Surg 135(7):849-853

6. Paquette S, Lin JC (2019) Outpatient telemedicine program in vascular surgery reduces patient travel time, cost, and environmental pollutant emissions. Ann Vasc Surg 59:167-172

7. Irarrazaval MJ, Inzunza M, Munoz R, et al. (2020). Telemedicine for postoperative follow-up, virtual surgical clinics during COVID-19 pandemic. Surg Endosc.

8. Nikolian VC, Williams AM, Jacobs BN et al (2018) Pilot study to evaluate the safety, feasibility, and financial implications of a postoperative telemedicine program. Ann Surg 268(4):700-707

9. Poulose BK, Shelton J, Phillips S et al (2012) Epidemiology and cost of ventral hernia repair: making the case for hernia research. Hernia 16(2):179-183

10. Dabbas N, Adams K, Pearson K et al (2011) Frequency of abdominal wall hernias: is classical teaching out of date? JRSM Short Rep 2(1):5

11. McGillicuddy JW, Gregoski MJ, Weiland AK et al (2013) Mobile health medication adherence and blood pressure control in renal transplant recipients: a proof-of-concept randomized controlled trial. JMIR Res Protoc 2(2):e32

12. Bednarski BK, Slack RS, Katz M et al (2018) Assessment of ileostomy output using telemedicine: a feasibility trial. Dis Colon Rectum 61(1):77-83

13. Armstrong K, Coyte P, Semple J (2015) The effect of mobile app follow-up care on the number of in-person visits following ambulatory surgery: a randomized control trial. Stud Health Technol Inform 216:894

14. Williams AM, Bhatti UF, Alam HB et al (2018) The role of telemedicine in postoperative care. Mhealth 4:11

15. Gunter RL, Chouinard S, Fernandes-Taylor S et al (2016) Current use of telemedicine for post-discharge surgical care: a systematic review. J Am Coll Surg 222(5):915-927

16. Weinstein RS, Lopez AM, Joseph BA et al (2014) Telemedicine, telehealth, and mobile health applications that work: opportunities and barriers. Am J Med 127(3):183-187

17. Ricci MA, Caputo M, Amour J et al (2003) Telemedicine reduces discrepancies in rural trauma care. Telemed J E Health 9(1):3-11

18. Urquhart AC, Antoniotti NM, Berg RL (2011) Telemedicine-an efficient and cost-effective approach in parathyroid surgery. Laryngoscope 121(7):1422-1425 
19. Lavrentyev V, Seay A, Rafiq A et al (2008) A surgical telemedicine clinic in a correctional setting. Telemed J E Health 14(4):385-388

20. Marcin JP, Shaikh U, Steinhorn RH (2016) Addressing health disparities in rural communities using telehealth. Pediatr Res 79(1-2):169-176
Publisher's Note Springer Nature remains neutral with regard to jurisdictional claims in published maps and institutional affiliations. 